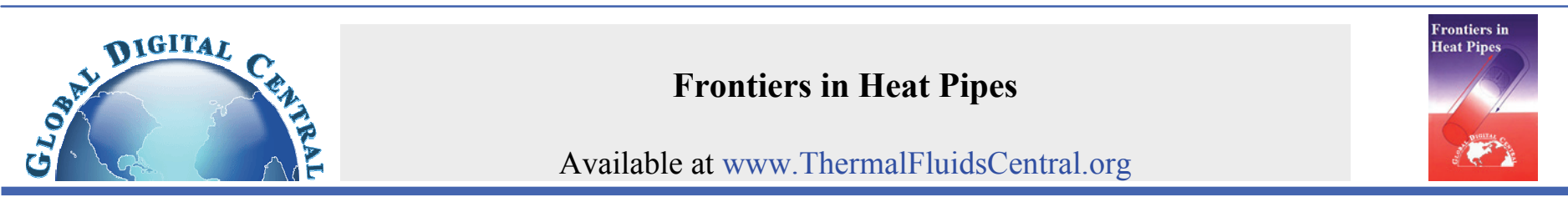

\title{
EFFECTS OF CAPILLARY AND GRAVITATIONAL FORCES ON PERFORMANCE OF AN OSCILLATING HEAT PIPE
}

\author{
Wei Shao, Yuwen Zhang* \\ Department of Mechanical and Aerospace Engineering, University of Missouri, Columbia, MO 65211, USA
}

\begin{abstract}
An advanced mathematical model of flow and heat transfer in an oscillating heat pipe (OHP) is proposed. The capillary and gravitational forces are included in the momentum equation of the liquid slug. Detailed numerical simulation is carried out to investigate the surface tension and gravity effects on the oscillatory flow and heat transfer in an OHP with different inner diameters and orientations. The results show that gravity effect hinders the performance of top heat mode OHP while aids the operation of bottom heat mode OHP. Comparisons between the cases with surface tension and without surface tension indicate that the effects of surface tension on the performance of OHP are negligible even for small inner diameter. The effect of inclination angle on the performance of OHP is investigated.

Keywords: Oscillating Heat Pipe, Capillary Force, Gravity
\end{abstract}

\section{INTRODUCTION}

The increased demanding on high performance required by the IT industry has raised thermal design challenges due to both increased heat dissipation from the CPU and higher heat density. Local hot spot heat fluxes within the CPU are exceeding $100 \mathrm{~W} / \mathrm{cm}^{2}$ causing the CPU temperature rise, malfunction and failure of CPU without a very effective heat transfer device. While conventional heat sinks or spreaders become severely inadequate at these high levels of heat fluxes, Oscillating (or Pulsating) Heat Pipe (OHP or PHP) shows promise to meet the next generation CPU thermal requirements with a low profile heat sink. The OHP is a new type of two-phase heat transfer devices, invented and patented in 1990's by Akachi (1994). Due to its potential heat transport capability, fast thermal response, simple structure and low cost of construction, the OHP will play a key role in the electronics cooling.

In the last decade, extensive experimental and theoretical works have been conducted to understand the mechanism of the OHP. Dobson and Harms (1999) developed a simple mathematical model to study the behavior of an OHP with an open-end. They simply assumed the heat transfer coefficients between heated pipe wall and the vapor and neglected the effect of surface tension and heat transfer between the liquid and its surroundings. Hosoda et al. (1999) investigated the formation of vapor plugs in a meandering closed loop heat transport device (MCL-HTD) with a simplified numerical model neglecting liquid film between the tube wall and the vapor plug and the effect of surface tension. Wong et al. (1999) presented a theoretical model of OHP operation based on the Lagrangian approach of slug flow in a serpentine tube. Zhang and Faghri (2002) analyzed thin film evaporation and condensation in the evaporator and condenser sections of an OHP with an open end. The results showed that the heat transfer in an OHP is mainly due to the exchange of sensible heat and phase- change heat transfer is the driving force of the oscillation. Liang and Ma (2004) presented a mathematical model describing the oscillation characteristics of slug flow in a capillary tube. It was demonstrated that the internal diameter, vapor plug size, and unit cell numbers determine the oscillation and capillary force, gravitational force, initial pressure distribution of the working fluid significantly affect the frequency and amplitude of oscillating motion in the capillary tube. Kiseev and Zolkin (1999) experimentally investigated the effects of acceleration and vibration on the performance of the unlooped OHP and the results indicated that the OHP operates successfully by various acceleration effects. By increasing the acceleration from $-6 g$ to $+12 g$ the evaporator temperature was increased by $30 \%$. Recently, the authors extended the modeling capability by including the effects of initial temperature, axial variation of surface temperature, and pressure drop at the bend (Shao and Zhang, 2011). It was demonstrated that the initial temperature has a very profound effect in oscillating motion and heat transfer performance.

The objective of this work is to study the effect of gravity and capillary force on the performance of an oscillating heat pipe.

\section{THEORETICAL MODEL}

Figure 1 shows the physical model of a U-shaped minichannel with its two ends sealed. It is considered as the building block of an OHP. The length of each evaporator section, which is located at the two ends of the pipe, is $L_{\mathrm{e}}$ and evaporator temperature is maintained at $T_{\mathrm{e}}$. The condenser section is located between two evaporation sections with a length of $\mathrm{L}_{\mathrm{c}}$. The temperature of the condenser section is maintained at $T_{\mathrm{c}}$. The length of the liquid slug is $L_{\mathrm{p}}$, which depends on the filling ratio. The displacement of the liquid slug is represneted by $x_{\mathrm{p}}$. When the liquid slug is exactly in the middle of the U-shaped miniature channel, $x_{\mathrm{p}}$ is zero. When the liquid slug shifts to the right side, $x_{\mathrm{p}}$ is positive; when it moves to the left side $x_{\mathrm{p}}$ is negative. Once the

\footnotetext{
*Corresponding author.Email: zhangyu@missouri.edu
} 
oscillation starts, the contact angles at both ends of the liquid slug will be different, depends on the direction of liquid slug flow.

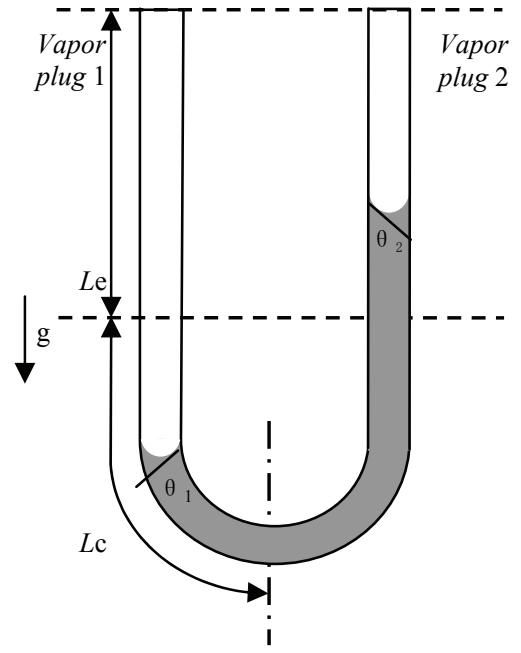

(a)

Vapor

Liquid

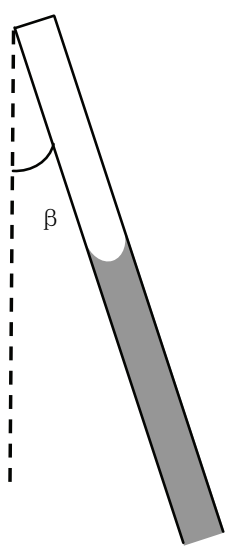

(b)
Fig. 1 Physical model

If the initial value of displacement $x_{\mathrm{p} 0}$ is positive (see Fig. 1(a)), the pressure of left vapor slug decreases condensation. Meanwhile, the right vapor plug increases due to evaporation of liquid film left behind by the liquid slug. The pressure difference between the two vapor plugs, in turn, causes the liquid slug moving to the left direction. After the displacement change its sign from positive to negative, the pressure difference will also change its sign to cause the liquid slug to move from left to right. The oscillation of the liquid slug can be sustained by alternative evaporation and condensation in the two vapor plugs. The following assumptions are made in order to model heat transfer and fluid flow in the heat pipe:

(1) The liquid is incompressible and the vapor is saturated and behaves as an ideal gas.

(2) Evaporative and condensation heat transfer coefficients are assumed to be constants

(3) Shear stress at the liquid-vapor interface is negligible.

(4) Heat conduction in the liquid slug is assumed to be onedimensional in the axial direction and exchange of heat between the liquid and wall is considered by a convective heat transfer coefficient.

(5) The U-shaped minichannel is assumed to be a straight pipe and the effect of pressure loss in bend is considered using an empirical correlation.

\subsection{Governing Equations for Oscillatory Flow}

The momentum equation for the liquid slug is:

$$
A L_{p} \rho_{l} \frac{d^{2} x_{p}}{d t^{2}}=\left[\left(p_{l, 1}-p_{l, 2}\right)-\Delta p_{b}\right] A-2 \rho_{l} g \cos \beta A x_{p}-\pi d L_{p} \tau_{p}
$$

where the liquid pressures are related to the vapor pressures by Laplace-Young equation:

$$
\begin{aligned}
& p_{v 1}-p_{l, 1}=\frac{2}{r} \sigma_{1} \cos \theta_{1} \\
& p_{v 2}-p_{l, 2}=\frac{2}{r} \sigma_{2} \cos \theta_{2}
\end{aligned}
$$

The pressure difference between the two ends of the liquid slug becomes:

$$
p_{l, 1}-p_{l, 2}=\left(p_{v 1}-p_{v 2}\right)-\frac{2}{r}\left(\sigma_{1} \cos \theta_{1}-\sigma_{2} \cos \theta_{2}\right)
$$

where the surface tension is function of temperature. For water, the surface tension can be expressed as:

$$
\sigma_{i}=\left[75.83-0.1477 \times\left(T_{v, i}-273.15\right)\right] \times 10^{-3} \mathrm{~N} / \mathrm{m}
$$

The contact angles at two ends depend on the direction of liquid slug movement. When the liquid slug velocity is positive $\left(v_{p}>0\right)$, the contact angles are $\theta_{1}=\theta_{\min }, \theta_{2}=\theta_{\max }$ where $\theta_{\min }$ and $\theta_{\max }$ are minimum and maximum contact angle. When the liquid slug velocity is negative $\left(v_{p}<0\right)$, the contact angle becomes $\theta_{1}=\theta_{\max }, \theta_{2}=\theta_{\min }$. The pressure loss at the bend can be found from Rohsenow et al (1985):

$$
\Delta p_{b}=\left\{\begin{array}{cc}
K \rho_{l} \frac{v_{p}^{2}}{2} & v_{p}>0 \\
-K \rho_{l} \frac{v_{p}^{2}}{2} & v_{p}<0
\end{array}\right.
$$

where $K$ is the pressure loss coefficient. The effect of gravity on the motion of the liquid slug has been included in the momentum equation. Depends on the orientation of the OHP, the gravity may either assist or drag the oscillatory flow. The shear stress is obtained from $\tau_{p}=c_{l} \rho v_{p}^{2} / 2$, where $c_{l}$ is the friction coefficient that depends on the Reynolds number of liquid slug.

The energy equations of the two vapor plugs are:

$$
\begin{aligned}
& \frac{d\left(m_{v 1} c_{v} T_{v 1}\right)}{d t}=c_{p} T_{v 1} \frac{d m_{v 1}}{d t}+p_{v 1} \frac{\pi}{4} d^{2} \frac{d x_{p}}{d t} \\
& \frac{d\left(m_{v 2} c_{v} T_{v 2}\right)}{d t}=c_{p} T_{v 2} \frac{d m_{v 2}}{d t}-p_{v 2} \frac{\pi}{4} d^{2} \frac{d x_{p}}{d t}
\end{aligned}
$$

The vapor plugs behave as an ideal gas, i.e.,

$$
\begin{aligned}
& p_{v 1}\left(L_{e}+x_{p}\right) \frac{\pi}{4} d^{2}=m_{v 1} R_{g} T_{v 1} \\
& p_{v 2}\left(L_{e}-x_{p}\right) \frac{\pi}{4} d^{2}=m_{v 2} R_{g} T_{v 2}
\end{aligned}
$$

Combining the above equations, the masses and temperatures of two vapor plugs are obtained as following:

$$
\begin{gathered}
m_{v 1}=\frac{\pi d^{2} p_{0}}{4 R T_{0}}\left(\frac{p_{v 1}}{p_{0}}\right)^{\frac{1}{\gamma}}\left(L_{e}+x_{p}\right) \\
m_{v 2}=\frac{\pi d^{2} p_{0}}{4 R T_{0}}\left(\frac{p_{v 2}}{p_{0}}\right)^{\frac{1}{\gamma}}\left(L_{e}-x_{p}\right) \\
T_{v 1}=T_{0}\left(\frac{p_{v 1}}{p_{0}}\right)^{\frac{(\gamma-1)}{\gamma}} \\
T_{v 2}=T_{0}\left(\frac{p_{v 2}}{p_{0}}\right)^{\frac{(\gamma-1)}{\gamma}}
\end{gathered}
$$

The initial condition of the U-shaped minichannel in this study is chosen to be identical to the reference state of this system, i.e.,

$$
\begin{gathered}
x_{p}=x_{p 0}, t=0 \\
p_{v 1}=p_{v 2}=p_{0}, t=0 \\
T_{v 1}=T_{v 2}=T_{0}, t=0 \\
m_{v 1}=\frac{\pi d^{2} p_{0}}{4 R_{g} T_{0}}\left(L_{e}+x_{p 0}\right) \\
m_{v 2}=\frac{\pi d^{2} p_{0}}{4 R_{g} T_{0}}\left(L_{e}-x_{p 0}\right)
\end{gathered}
$$


The evaporation and condensation heat transfer in the left and right vapor plugs is related to the mass flux due to evaporation and condensation:

$$
\begin{aligned}
& Q_{i n, v 1}=\dot{m}_{e v p, v 1} h_{l v} \\
& Q_{o u t, v 1}=\dot{m}_{c o n, v 1} h_{l v} \\
& Q_{i n, v 2}=\dot{m}_{e v p, v 2} h_{l v} \\
& Q_{o u t, v 2}=\dot{m}_{c o n, v 2} h_{l v}
\end{aligned}
$$

Since the liquid slug is assumed to be incompressible, the entire liquid slug oscillates at the same velocity $v_{p}$. The temperature distribution in the liquid slug can be obtained by solving the energy equation for a liquid slug in a coordinate system that is moving with the liquid slug:

$$
\frac{1}{\alpha_{l}} \frac{\partial T_{l}}{\partial t}=\frac{\partial^{2} T_{l}}{\partial x_{l}^{2}}-\frac{h_{l s e n} \pi d}{k_{l} A}\left(T_{l}-T_{w}\right)
$$

where the thermophysical properties of the liquid slug is based on the mean temperature of the liquid slug. Equation (24) is subject to the following initial and boundary conditions

$$
\begin{gathered}
T=T_{0}, t=0,0<x_{l}<L_{p} \\
T=T_{v 1}, x_{l}=0 \\
T=T_{v 2}, x_{l}=L_{p}
\end{gathered}
$$

The wall temperature of the tube can be either $T_{e}$ or $T_{c}$, depending on the displacement of the liquid slug, i.e.,

When $x_{p}>0$,

$$
T_{w}=\left\{\begin{array}{cc}
T_{c}, & 0<x_{l}<L_{p}-x_{p} \\
T_{e}, & L_{p}-x_{p}<x_{l}<L_{p}
\end{array}\right.
$$

When $x_{p}<0$,

$$
T_{w}=\left\{\begin{array}{cc}
T_{e}, & 0<x_{l}<\left|x_{p}\right| \\
T_{c}, & \left|x_{p}\right|<x_{l}<L_{p}
\end{array}\right.
$$

Since the Reynolds number of the liquid slug varies in a wide range that covers laminar, transition and turbulent flow, the heat transfer coefficient $h$ of the liquid slug varies periodically. For laminar regime, $\left(\operatorname{Re}=\rho_{l} v_{p} d / \mu_{l}<2200\right)$, the convective heat transfer problem is considered to be thermally developing Hagen-Poiseuille flow problem. With the effect of axial step variation of surface temperature considered, the Nusselt number is obtained using method of superposition Rohsenow et al. (1985). In contrast to the existing models in the literature that assumed constant Nusselt number, variation of Nusselt number along the length of the liquid slug is taken into consideration. In the transition and turbulent regions, the following empirical correlations are used (Gnielinski, 1975):

$$
\begin{gathered}
N u=0.012\left(|\operatorname{Re}|^{0.87}-280\right) \operatorname{Pr}_{m}{ }^{0.4}\left(\frac{\operatorname{Pr}_{m}}{\operatorname{Pr}_{w}}\right)^{0.11}\left[1+\left(\frac{d}{L_{p}}\right)^{\frac{2}{3}}\right], \\
N u=0.0236|\operatorname{Re}|^{0.8} \operatorname{Pr}_{m}{ }^{0.43}\left(\frac{\operatorname{Pr}_{m}}{\operatorname{Pr}_{w}}\right)^{0.25},|\operatorname{Re}|>10000
\end{gathered}
$$

The sensible heat transfer into and out from the liquid slug can be obtained by integrating the heat transfer over the length of the liquid slug, i.e.

$$
Q_{i n, s, l}=\left\{\begin{array}{l}
\int_{L_{p}-x_{p}}^{L_{p}} \pi d h\left(T_{e}-T_{l}\right) d x_{l}, x_{p}>0 \\
\int_{0}^{\left|x_{p}\right|} \pi d h\left(T_{e}-T_{l}\right) d x_{l}, x_{p}<0
\end{array}\right.
$$

$$
Q_{o u t, s, l}=\left\{\begin{array}{l}
\int_{0}^{-x_{p}} \pi d h\left(T_{l}-T_{c}\right) d x_{l}, x_{p}>0 \\
\int_{\left|x_{p}\right|}^{L_{p}} \pi d h\left(T_{l}-T_{c}\right) d x_{l}, x_{p}<0
\end{array}\right.
$$

where the sensible heat transfer coefficient can be obtained from $h=N u k_{l} / d$

The sensible heat transfer into and out from the liquid slug can be obtained by integrating the heat transfer over the length of the liquid slug (Kays et al., 2005).

\section{NUMERICAL SOLUTION}

The governing equations in the above physical model can be solved numerically. An explicit finite difference scheme is employed to solve the governing equations of the liquid slug and vapor plug. An implicit scheme with uniform grid Patankar (1980) is employed to solve transient heat transfer in the liquid slug. The total number of grids chosen for the liquid slug is 300 and doubling the number of grids does not change the results. The results of each time-step can be obtained by following the numerical procedure outlined below:

1) Assume the temperatures of the two vapor plugs $T_{v 1}$ and $T_{v 2}$, and calculate thermal and physical properties of liquid according to $T_{l}$

2) Solve for vapor pressures, $p_{v 1}$ and $p_{v 2}$, from eqs. (9) and (10).

3) Solve for $x_{p}$ from eqs. (1) and (6).

4) Obtain the new masses of the two vapor plugs $m_{v 1}$ and $m_{v 2}$, by accounting for the change of vapor masses from eqs. (11) and (12).

5) Calculate the pressure of the two vapor plugs, $p_{v 1}$ and $p_{v 2}$, from eqs. (9) and (10).

6) Solve for $T_{v 1}$ and $T_{v 2}$ from eqs. (13) and (14).

7) Compare $T_{v 1}$ and $T_{v 2}$ obtained in Step 6 with assumed values in step 1. If the differences meet the small tolerance, then go to the step 8; otherwise, the above procedure is repeated until a converged solution is obtained.

8) Obtain heat transfer coefficient through eqs. (30)to (31).

9) Solve for liquid temperature distribution from eq. (24) and calculate $Q_{s}$.

10) Use eqs. (20) and (23) to calculate $Q_{\text {latent }}$

After the time-step independent test, it was found that the timestep independent solution of the problem can be obtained when timestep is $\Delta t=1 \times 10^{-4}$, which is used in all numerical simulations.

\section{RESULTS AND DISCUSSION}

The parameters of the miniature channel are: $L_{e}=0.1 \mathrm{~m}, L_{c}=0.2 \mathrm{~m}, L_{p}$ $=0.2, d=0.001 \mathrm{~m}, T_{e}=123.4{ }^{\circ} \mathrm{C}, T_{c}=20^{\circ} \mathrm{C}$. The initial pressures of the vapor plugs are $p_{v 0}=31164.64 \mathrm{~Pa}$ and the initial temperature of the vapor plug is $T_{v 0}=70{ }^{\circ} \mathrm{C}$. The heat transfer coefficient at the heating and cooling sections are is $h_{e}=h_{c}=200 \mathrm{~W} / \mathrm{m}^{2}-\mathrm{K}$. The maximum and minimum contact angels, $\theta_{\max }$ and $\theta_{\min }$, between water and cupper wall are $85^{\circ}$ and $33^{\circ}$, respectively. Unless stated otherwise, the above conditions remain the same for each case. 


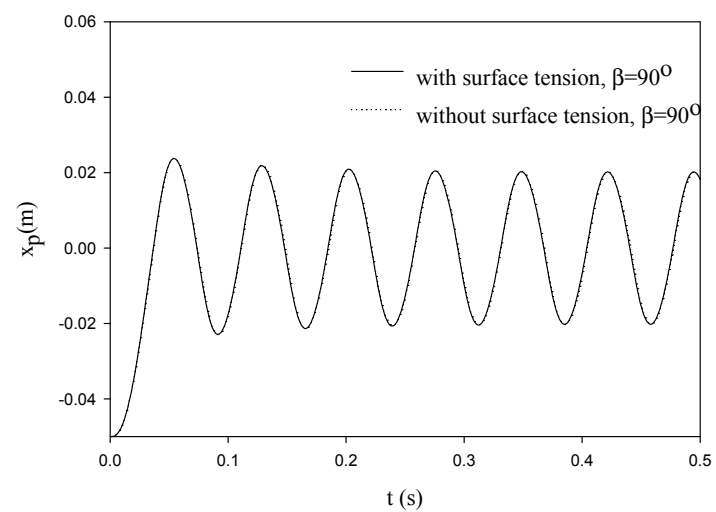

(a) Displacement of liquid slug

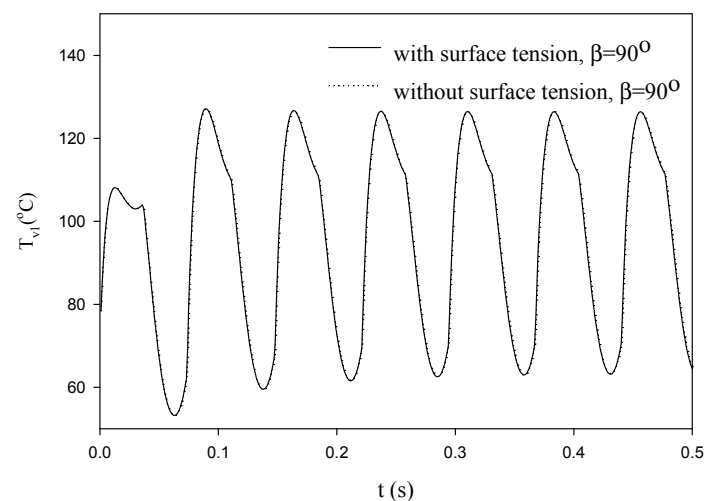

(b) Vapor plug temperatures

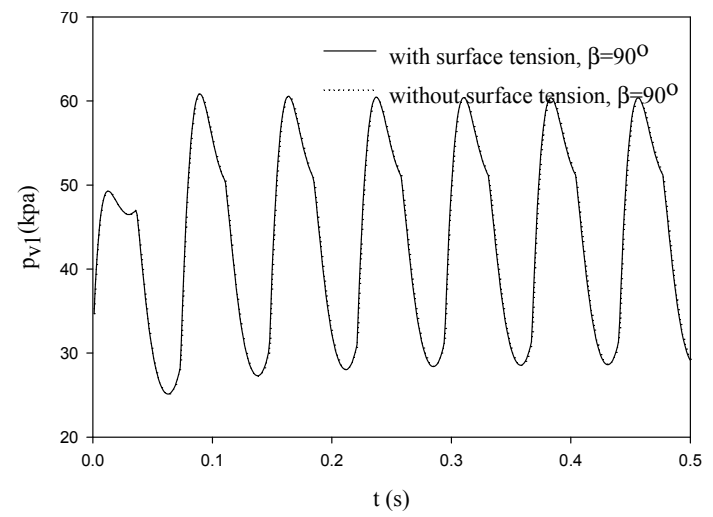

(c) Vapor plug pressure

Fig. 2 Comparison of with surface tension and without surface tension at $\beta=90^{\circ}$

Figure 2(a) shows the comparison of liquid slug displacements for the cases with and without surface tension with $\beta=90^{\circ}$ and $\mathrm{d}=0.001$ $\mathrm{mm}$. As can be seen, there is no apparent difference between the displacements for the cases with and without surface tension since the capillary force is relatively small compared with the vapor pressure difference. Figure 2(b) shows the comparison of left vapor plug temperatures for the cases with and without surface tension. Similar to the trend on liquid slug displacement, there is no effect from surface tension. The comparison of the pressure variations of the left vapor plug for the cases with and without surface tension is shown in Fig. 2 (c). Once again, the vapor plug pressures exhibited the similar trend as the vapor plug temperatures. Therefore, one can conclude that capillary force has little effect on the oscillatory flow and heat transfer of an
OHP. Although the capillary force is the necessary for the formation of liquid slug, the performance of the OHP is not affected by the surface tension. The effect of capillary force on the heat transfer was also computed and no noticeable effect was observed.

Figure 3(a) shows the comparison of the displacement of liquid slug for five potential orientations (see Fig. 1(b)):

(1) $\beta=0^{\circ}$ (evaporator above condenser)

(2) $\beta=45^{\circ}$ (evaporator above condenser)

(3) $\beta=90^{\circ}$ (horizontally)

(4) $\beta=135^{\circ}$ (condenser above evaporator)

(5) $\beta=180^{\circ}$ (condenser above evaporator)

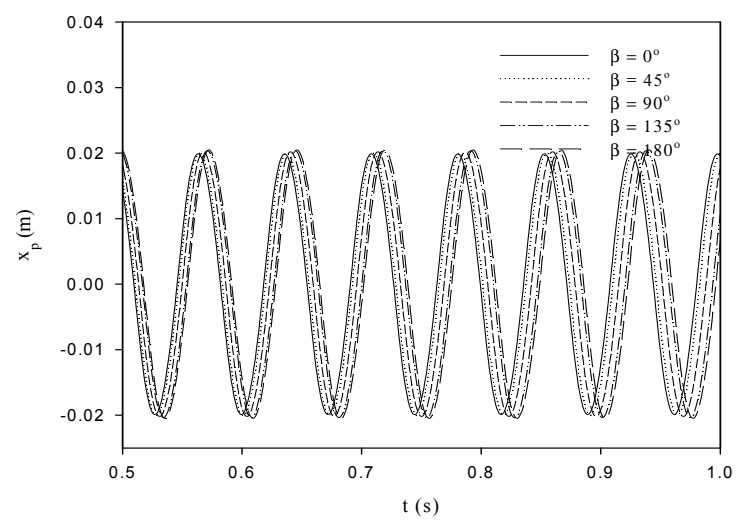

(a) Displacement of liquid slug

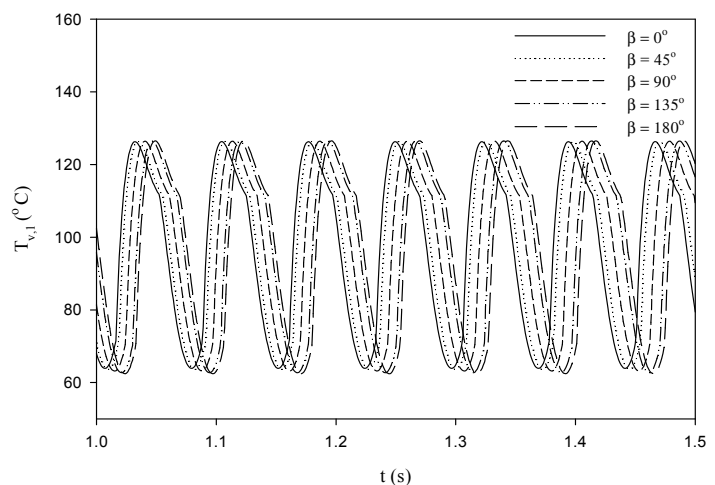

(b) Vapor plug temperatures

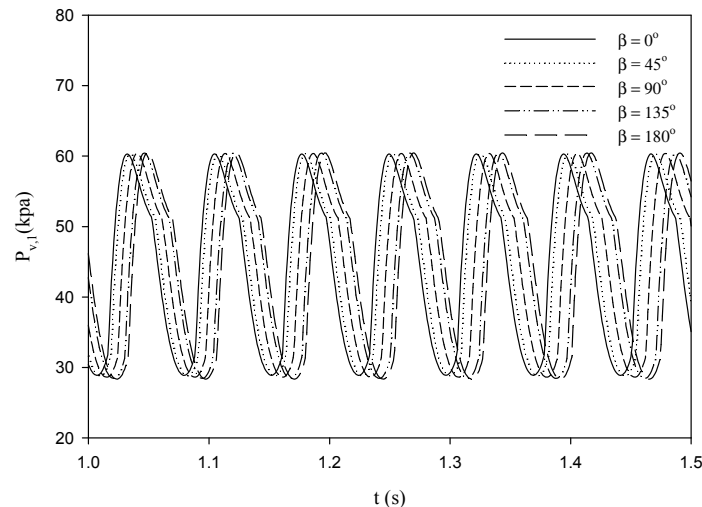

(c) Vapor plug pressure

Fig. 3 Comparison of the oscillations of different inclination angles 
As $\beta$ increases from $0^{\circ}$ to $180^{\circ}$, the gravitational acceleration decreases from $+\mathrm{g}$ to $-\mathrm{g}$. As can be seen from this comparison, at other condition being equal, there are an increase of the amplitude and a decrease of the frequency of the oscillation when $\beta$ increases. The change of amplitude of the liquid slug displacement is so small but the phase is much more delayed as $\beta$ increases. The frequency of oscillation for the case of $\beta=180^{\circ}$ is decreased compared with that of the case with $\beta=0^{\circ}$. Figure 3 (b) shows the comparison of left vapor plug temperatures in five different orientations. Similar to the trend on liquid slug displacement, there is a delay in the phase of the vapor temperature with increasing $\beta$. The frequency of the temperature oscillation is decreased as $\beta$ increasese. Comparison of the pressure variations of the left vapor plug for five different orientation angles is shown in Fig. 3 (c). It can be seen that the vapor plug pressures in five different orientations exhibited the similar trend as the vapor plug temperatures. Therefore, one can conclude that as $\beta$ increases, the frequencies of displacement, vapor plug temperature and pressure decrease, while the amplitude slightly increases.

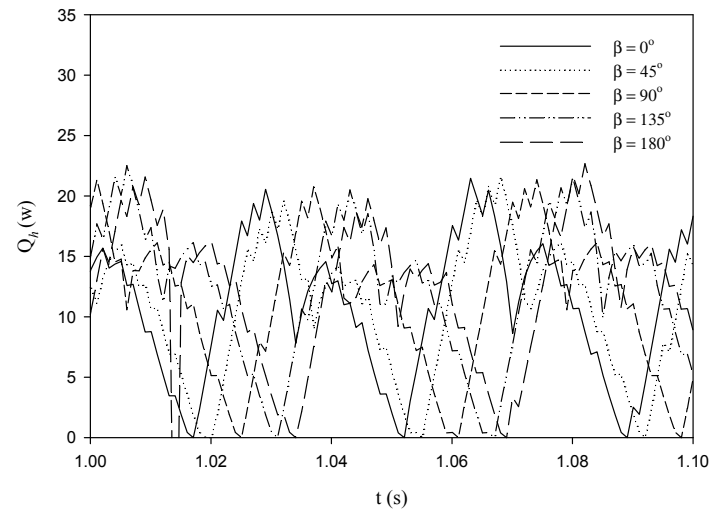

(a) Sensible heat transfer

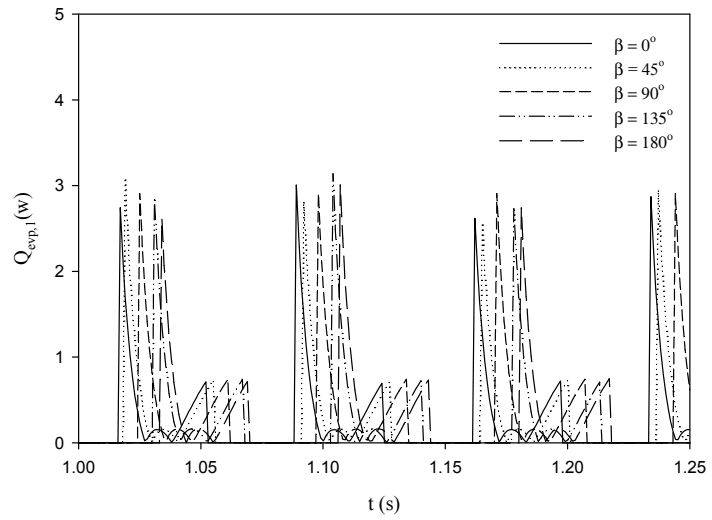

(b) Latent heat transfer

Fig. 4 Comparison of heat transfer of different inclination angles

Fig. 4 (a) shows the comparison of sensible heat transfer transferred into the liquid for five orientations. There is a delay of the phase and a slight increase of the of sensible heat transfer in and out of the liquid as $\beta$ increases. Due to enhanced oscillatory motion of the liquid slug, the average sensible heat transfer rate is increased from $10.95 \mathrm{~W}$ with $\beta=0^{\circ}$ to $11.64 \mathrm{~W}$ with $\beta=180^{\circ}-$ a $6.3 \%$ increase. The comparison of latent heat transfer for five different orientations is shown in Fig. 4(b). It is seen that the delay of the phase of the oscillation also results the delay of the phase of evaporation heat transfer but the amplitude of evaporation heat transfer does not increase much. Due to gravity effect, the average evaporation heat transfer increases from $0.49 \mathrm{~W}$ to $0.50 \mathrm{~W}-$ a $2 \%$ increase. Thus, the effect of gravitational force on the performance of the OHP is very insignificant.

The effect of diameter on the performance of OHP is studied next. Figure 5(a) shows the comparison of the displacement for the cases of five different diameters. As can be seen, at other condition being equal, there are an apparent decrease of the amplitude and a increase of the frequency of the oscillation when the inner diameter decreases together. It can be seen that, the change of amplitude of the liquid slug displacement is apparent and the phase is much more advanced. The amplitude of oscillation for the cases with $\mathrm{d}=0.001 \mathrm{~mm}$ is decreased compared with that of the case with $d=0.003 \mathrm{~m}$. Figure $5(\mathrm{~b})$ shows the comparison of left vapor plug temperatures for five different diameters. With increasing diameter, the amplitude of temperature oscillation decreases and the phase of oscillation advances. Figure 5(c) shows the comparison of the pressure variations of the left vapor plug in five different diameters. The vapor plug pressures in five different orientations exhibited the similar trend as the vapor plug temperatures.

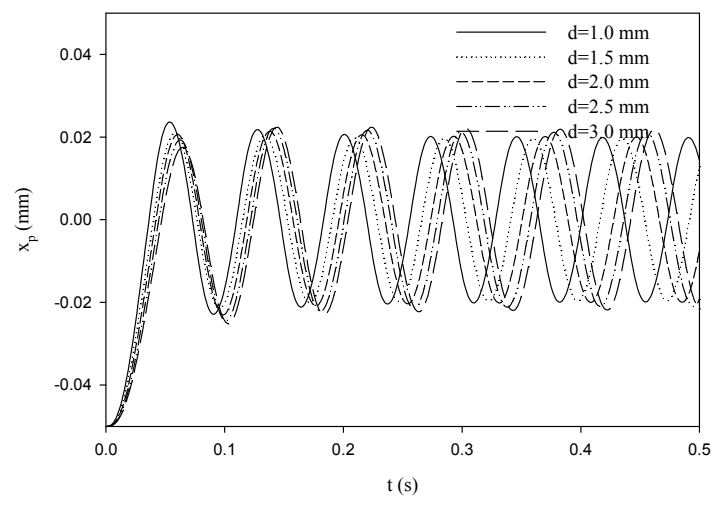

(a) Displacement of liquid slug

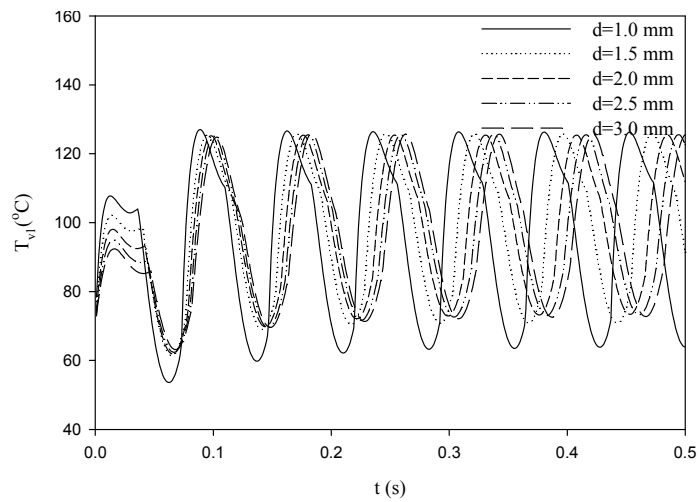

(b) Vapor plug temperatures

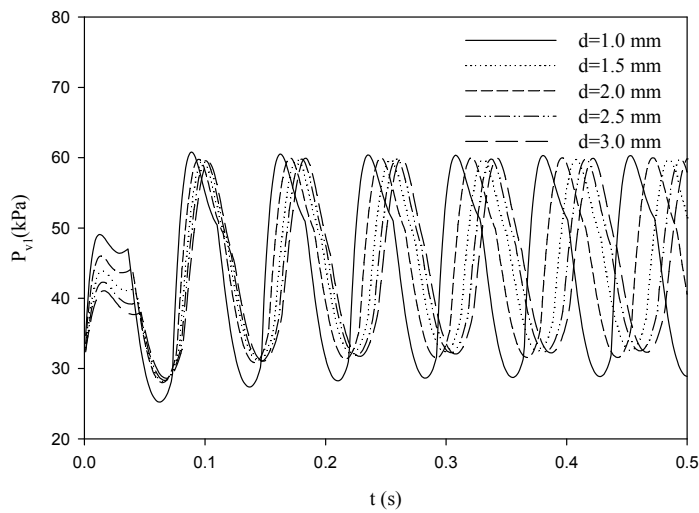

(c) Vapor plug pressure

Fig. 5 Comparison of the oscillations for different inner diameters 


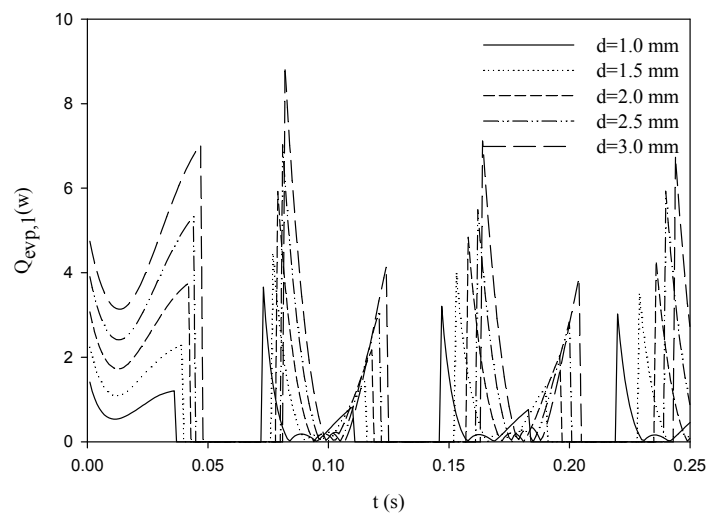

(a) Sensible heat transfer

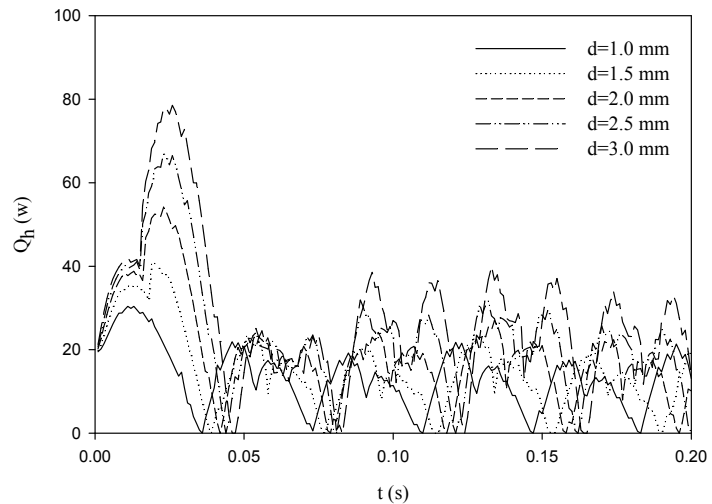

(b) Latent heat transfer

Fig. 6 Comparison of heat transfer for different inner diameters

Figure 6(a) shows the comparison of latent heat transfer for different inner diameters. The latent heat transfer increases as the inner diameter increases. Figure 6(b) shows the comparison of the sensible heat transfer for different inner diameters of OHP. There is an increase of the sensible heat transfer when the inner diameter increases. Although increasing the inner diameter can enhance the heat transfer, the inner diameter must be small enough to ensure formation of liquid slugs in the OHP.

\section{CONCLUSIONS}

Capillary and gravitational effects on oscillatory flow and heat transfer of an oscillating heat pipe are fully investigated by comparing displacement of liquid slug, temperature and pressure of vapor plugs, as well as latent and sensible heat transfer. It is concluded that the effect of surface tension on the oscillation can be neglected. The gravitational force has more effect on the frequency of the oscillation than the amplitude. The bottom-heating mode operates most effectively. As the inner diameter of the OHP increases, the heat transfer capability is improved.

\section{ACKNOWLEDGEMENTS}

Support for this work by the U.S. National Science Foundation under grant number CBET-1066917 is gratefully acknowledged.

\section{NOMENCLATURE}

$\begin{array}{ll}A & \text { area, } \mathrm{m}^{2} \\ c_{p} & \text { specific heat at constant pressure, } \mathrm{J} / \mathrm{kg}-\mathrm{K}\end{array}$

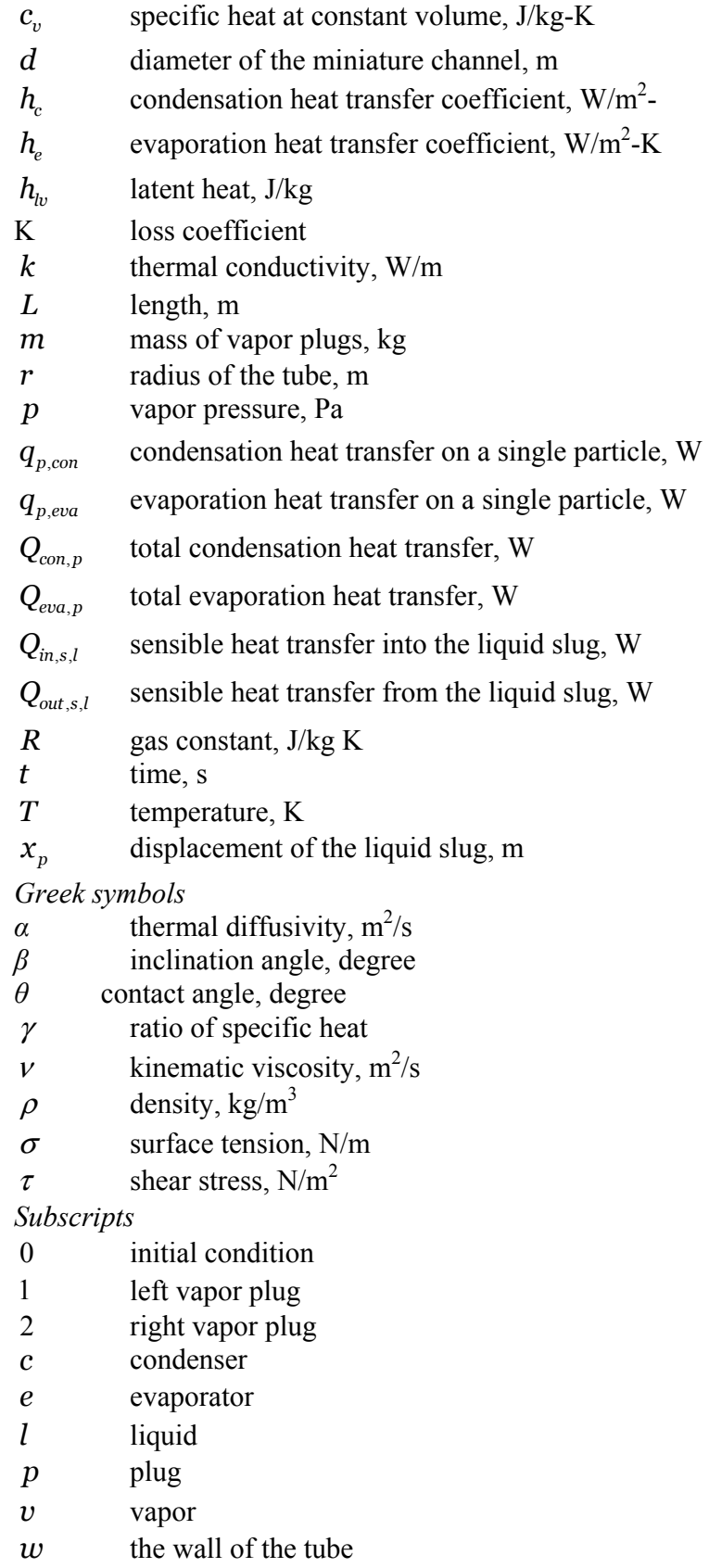

\section{REFERENCES}

Akachi, H., Looped Capillary Heat Pipes, Japanese Patent, No. Hei697147, 1994

Dobson, R.T., and Harms, T.M., Lumped Parameter Analysis of Closed and Open Oscillatory Heat Pipe, Proceedings of the 11th International Heat Pipe Conference, Tokyo, Japan, 1999, pp. 137-142

Gnielinski, V., 1975, Neue Gleichungen für den Wärme- und den Stoffübergang in turbulent durchströmten Rohren und Kanälen, Forschung im Ingenieurwesen, 41(1), pp. 8-16 http://dx.doi.org/10.1007/BF02559682

Hosoda, M., Nishio, S., and Shirakashi, R., Meandering closed-loop heat-transfer tube (propagation phenomena of vapor plug), Proceedings of the $5^{\text {th }} A S M E / J S M E$ Joint Thermal Engineering Conference, March 15-19, San Diego, CA, 1999.

Kays, W.M., Crawford, M.E., and Weigand, B., Convective Heat Transfer, $4^{\text {th }}$ ed., McGraw-Hill, New York, 2005, Chap. 8. 
Kiseev, V.M., and Zolkin, K.A.,1999, "The Influence of Acceleration on the Performance of Oscillating Heat Pipe," Proceedings of $11^{\text {th }}$ International Heat Pipe Conference, Tokyo, Japan, pp. 154-158.

Liang, S.B. and Ma, H.B., 2004, Oscillating motions of slug flow in capillary tubes, International Comm. Heat and Mass Transfer, 31(3), 365-375

http://dx.doi.org/10.1016/j.icheatmasstransfer.2004.02.007

Patankar, S.V., Numerical Heat Transfer and Fluid Flow, Hemisphere, New York, 19804, Chap. 2

Rohsenow, W.M., Hartnett, J.P. and Ganic, E.N., 1985, Handbook of Heat Transfer Fundamentals, $2^{\text {nd }}$ ed., McGraw-Hill, New York.
Shao, W., and Zhang, Y., 2011, "Thermally-Induced Oscillatory Flow and Heat Transfer in an Oscillating Heat Pipe," Journal of Enhanced Heat Transfer, 18(3), 177-190. http://dx.doi.org/10.1615/JEnhHeatTransf.v18.i3

Wong, T.N., Tong, B.Y., Lim, S.M., Ooi, K.T., Theoretical modeling of pulsating heat pipe, Proceedings of the $11^{\text {th }}$ International Heat Pipe Conference, Tokyo, Japan, 1999

Zhang, Y., and Faghri, A., "Heat transfer in a Pulsating Heat Pipe with Open End," International Journal of Heat and Mass Transfer, 45(18), 2501-2508

http://dx.doi.org/10.1016/S0017-9310(01)00348-9 